\title{
Prenatal maternal cortisol levels and infant behavior during the first 5 months
}

\author{
Carolina de Weerth ${ }^{\mathrm{a}, *}$, Yvonne van Hees ${ }^{\mathrm{a}}$, Jan K. Buitelaar ${ }^{\mathrm{b}}$ \\ ${ }^{\mathrm{a} C h i l d}$ and Adolescent Psychiatry, University Medical Center Utrecht, \\ HP A01.468-Postbox 85500, 3508 GA Utrecht, The Netherlands \\ ${ }^{\mathrm{b}}$ Department of Psychiatry, University Medical Center Nijmegen, HP 333-Postbox 9101, \\ 6500 HB Nijmegen, The Netherlands
}

Accepted 10 July 2003

\begin{abstract}
Background and aims: Research on both animals and humans is providing more and more evidence that prenatal factors can have long-term effects on development. Most human studies have examined the effects of prenatal stress on birth outcome (i.e. shorter pregnancies, smaller infants). The few studies that have looked at the infants' later development have found prenatal stress to be related to more difficult temperament, behavioral/emotional problems and poorer motor/cognitive development. In this paper, we have examined links between late pregnancy cortisol levels and infant behavior during the first 5 months of life. Study design and subjects: Seventeen mothers and their healthy, full-term infants participated in this prospective, longitudinal study. The mothers' cortisol was determined in late pregnancy. The infants' behavior was videotaped during a series of bath sessions at the home: at 1, 3, 5, 7, 18 and 20 weeks of age. The mothers filled in temperament questionnaires (ICQ) in postnatal weeks 7 and 18. Results and conclusions: The infants were divided into two groups based on their mothers' late pregnancy cortisol values: high and low prenatal cortisol groups. A trend was found for the high cortisol infants to be delivered earlier than the low cortisol group. Furthermore, the behavioral observations showed the higher prenatal cortisol group to display more crying, fussing and negative facial expressions. Supporting these findings, maternal reports on temperament also showed these infants to have more difficult behavior: they had higher scores on emotion and activity. The differences between the infants were strongest at the youngest ages (weeks 1-7).

(C) 2003 Elsevier Ireland Ltd. All rights reserved.
\end{abstract}

Keywords: Prenatal; Maternal cortisol level; Infant behavior

* Corresponding author. Tel.: +31-30-2509425; fax: +31-30-2505487.

E-mail address: C.deWeerth@psych.azu.nl (C. de Weerth). 


\section{Introduction}

Almost 30 years ago, Stott [1] (p. 770) wrote: “The prenatal determinants of individual differences remain a 'grey area' of modern science, yet their importance is incontestable. . knowledge of these determinants is central to our understanding of human development...". In the last decades, many researchers have indeed been looking more closely at the effects of prenatal factors on development. The result is that there is more and more evidence that shows that prenatal influences can have long-term and even permanent effects on development. Among others, Barker [2] has repeatedly reported how variations within the range of normal birth weights can be important predictors for risk of cardiovascular disease at adult age. Similarly, psychiatric epidemiological research points in the direction of prenatal factors such as stress and malnutrition for the appearance of schizophrenia [3].

Hormones that are secreted by the adrenal glands, i.e. products of the HPA-axis (hypothalamic-pituitary-adrenal axis), appear to have an especially important influence on brain development during the fetal period. These early hormonal effects are called 'programming' influences because the structure of the brain is being laid. Little is known about the early programming effects of the HPA-axis in humans. The HPAaxis is essential for coping with stress. When an organism is confronted with a stressor, a quick increase and then decrease of cortisol will characterize an adequate physiological stress reaction. On the other hand, abnormal reactivity of the HPA-axis has been found to be a vulnerability factor for psychopathology later in life, particularly for depression [4].

Most studies on prenatal stress have examined its effects on birth outcome. In general, stress during pregnancy has been related to shorter gestations and prematurity, and to smaller birth weights, but links have also been found to congenital malformations, smaller head circumferences, reduced neonatal neurological optimality, etc. (see for example Refs. [5-10] and for reviews Refs. [11,12]). Also, in an interesting study that examined behavior and cognition before birth, Wadhwa et al. [13] found that fetuses of mothers with elevated CRH levels did less well in in vivo studies of learning in fetuses.

Nonetheless, there are few studies of sufficient length that investigate the influence of prenatal stress on the HPA-axis and the behavior and development of the child after birth. Susman et al. [14], for example, established a relation between low levels of maternal hormones (cortisol among others) at 3 months of pregnancy and greater aggressiveness in the children at 3 years of age, which is in line with earlier findings on the HPA-axis and antisocial behavior [15-17]. O'Connor et al. [18] found prenatal maternal anxiety to predict behavioral/emotional problems in a very large sample of children at 4 years of age. Also, Huizink et al. $[19,20]$ found prenatal maternal stress, psychosocially and endocrinologically measured in a prospective study, to be related to poorer mental and motor development of the infant. Graham et al. [21] showed infants of depressed mothers to be more irritable, have growth delays, higher neonatal levels of cortisol and norepinephrine, poorer motor and cognitive development, etc., with effects present at least till the age of 3 years. Finally, earlier studies on the effects of stress during pregnancy also suggest long-term influences on offspring, such as children 
having poorer health, developmental delays, and showing more behavioral disturbances $[1,22]$.

However, the interaction between the various psychobiological factors during pregnancy is subtle and highly complicated, and much more research is needed to fill in our gaps of knowledge. This paper shows the results of a prospective, longitudinal study on the effects of prenatal stress. Specifically, we investigated the links between late pregnancy cortisol levels and infant behavior during a routine mother-infant interaction. Maternal behavior and infant temperament were also scored and the infants were followed till the age of 20 weeks in order to see whether behavioral changes occurred in the first months.

Based on most of the findings in the literature, we hypothesized that infants born to mothers with higher cortisol levels would be more irritable during the interactions and have a more difficult temperament than infants of mothers with lower levels of the hormone. Because the mothers in the study were highly educated and were in stable social-economical situations, we did not expect important differences in maternal behavior between the higher and lower cortisol groups. Finally, given that the postnatal environment was expected to be relatively normal and stable for all infants, the effects on behavior were expected to be strongest in the early postnatal period.

\section{Methods}

\subsection{Subjects}

The subjects were healthy women, which were recruited in their 30th to 35th weeks of pregnancy, through midwife practices in Utrecht. During the regular checkups, the midwives gave out information folders to all healthy patients, which had uncomplicated pregnancies and were in the required pregnancy stadium. Out of the 20 women who applied to participate, 3 subjects did not participate in the study because of private circumstances or preterm delivery. The study was approved by the Medical Ethical Committee of our hospital and the mothers gave informed consent to the work.

Altogether 17 women, aged 26-38 years, participated in the study. Four of the women had completed their secondary education and 13 had tertiary or university degrees. Of the 17 infants, 7 males and 10 females, 7 were firstborns, 8 were second children, 1 was a third child and 1 a fourth. All infants were the result of full-term, singleton pregnancies with uncomplicated deliveries. Their Apgar scores at birth were: 8-10 after 1 min and 9-10 after $5 \mathrm{~min}$. All the infants but one belonged to two parent families.

\subsection{Study design}

The mothers were followed from week 36 of pregnancy till week 20 after the delivery. During this period, data were collected in three main areas: cortisol, behavior and temperament. This was done during seven home-visits that were carried out individually by two female research assistants, i.e. each one had her own mother-infant pairs. The 
schedule in Fig. 1 shows exactly when each assessment moment took place and which data were collected at that time.

\subsection{Behavioral observations}

During six home visits (see Fig. 1), the mother-infant pair was videotaped while the mother gave the child a bath (which included undressing, dressing and sometimes taking temperature and weighing). This bath situation was chosen for three reasons: (1) It is a 'standard situation' which is fairly comparable across mothers and across the chosen ages; (2) it is a situation with a large amount of mother-infant interaction in a relatively short time; and (3) it takes place at home, thus providing the basis for more natural motherinfant interactions than in lab situations. The average bath session took about $20 \mathrm{~min}$. After reaching a sufficient level of reliability, the two research assistants scored the videotapes with The Observer ${ }^{\circledR}$ (Noldus Information Technology, Wageningen, The Netherlands). The assistants were blind to the mothers' prenatal stress status during the whole scoring period.

The ethogram, that was designed for this specific study, consisted of six duration systems (duration and frequency of behaviors):

- state infant:

- awake (eyes open, small to large amount of motor activity)

- drowsy/asleep (eyes closed, or seldom open, small amount or no motor activity)

- pacifier mother (finger/dummy):

- pacifier in (infant has pacifier/dummy/mother's finger in mouth)

- pacifier out (infant does not have pacifier/dummy/mother's finger in mouth)

- physical contact mother-infant:

- care (mother handles infant with caretaking purpose only: changing clothes, washing, drying, combing hair, etc.)

- care-minus (same as 'care', except that mother restricts the movements of one or more of the infant's limbs)

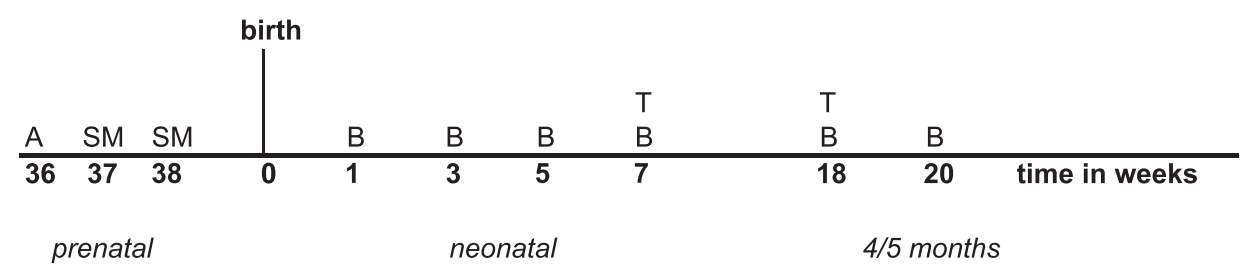

$\begin{array}{ll}\text { B } & \text { bath session } \\ \text { SM } & \text { saliva mother } \\ \text { A } & \text { social medical anamnesis } \\ \text { T } & \text { temperament questionnaire }\end{array}$

Fig. 1. Study design with assessment moments and type of data collected. 
- care-plus (same as 'care', except that "loving" activities take place at the same time: washing and rocking infant in bath, massaging the infant with oil/lotion, washing and stroking/cuddling infant, etc.)

- love (activities with no care-taking purpose, "loving" handling of the infant by the mother: rocking in bath, cuddling, stroking, etc.)

- play (playful contact between mother and infant: tickling, "biting" playfully, giving the infant a toy, playing together)

- no contact (no physical contact between mother and child for at least $3 \mathrm{~s}$ )

- facial expression baby:

- positive (smiling or laughing)

- bland (neutral, neither positive nor negative)

- negative (corners of the mouth are directed downwards)

- visual behavior baby:

- open (eyes are open)

- closed (eyes are closed, usually during sleeping or crying)

- vocalization baby:

- happy (positive or neutral vocalizations for at least $3 \mathrm{~s}$ : cooing, babbling, etc.)

- fussing (vocalizations of a clearly negative affect, usually accompanied by a negative facial expression, lasting at least $3 \mathrm{~s}$ : whining, fretting, etc.)

- crying (at least three definite weeping vocalizations, with a continuous rhythm and a negative facial expression)

- no vocalization (infant is silent for at least $3 \mathrm{~s}$ )

and two event systems (frequency of behaviors):

- reflexes baby (each of these behaviors counts as one):

- startle (Moro reflex; arms are widely stretched and hands make grasping movements, usually when infant goes in/out of bath or when picked up or laid down)

- hiccups

$\circ$ yawn

- sneeze

- kiss mother

- kiss (mother kisses infant, each kiss counts as one)

Reliability analyses. After training extensively on other videotapes, both research assistants scored five randomly chosen videotapes. The mean percentage agreement over the behavioral categories was $77.1 \%$ and the mean Cohen's $\kappa$ [23] was 0.74 .

\subsection{Saliva collection and analysis}

The mothers collected their saliva themselves in small pots when awakening in the morning. The samples were kept at $-18{ }^{\circ} \mathrm{C}$ until the moment of cortisol assessment at the Laboratory of Endocrinology of the University Medical Center of Utrecht. The saliva was first decanted into small glass tubes and $2 \mathrm{ml}$ was used for the assay. Cortisol levels were 
determined by radioimmunoassay (RIA) after ether extraction and chromatography on Celite columns.

\subsection{Questionnaires}

Social-medical-anamnesis: Interview about health and SES of both parents, and information about the pregnancy, the delivery and the Apgar score of the infant after 1 and $5 \mathrm{~min}$. It was carried out by the research assistant during the first and second home visit, thus at 36 weeks of pregnancy and 1 week post-delivery.

Temperament questionnaire: Infant Characteristics Questionnaire (ICQ [24]).

It was completed twice by the mothers: in postnatal weeks 7 and 18. It consists of 30 questions in three different areas. Higher scores in each of these categories mean that the infant displays more difficult behavior in that area:

- emotion: questions about frequency of crying and agitation during the day, if it is easy or difficult to console the infant when he/she is crying or agitated, if the infant likes to play by him/herself.

- adaptation: questions about the infant's first reaction to strangers, to new surroundings, to holidays/visits and the ability to adapt to unexpected changes in the daily pattern of the infant.

- activity: questions about frequency of being excited/active, if the infant is often in a happy/excited mood and if the infant likes to play and how often he or she plays during the day.

\subsection{Missing data}

Because of inevitable circumstances (for example technical problems with the video camera, incomplete questionnaires, delivery before pregnancy week 38 , etc.), not all data could be obtained. For each data assessment point, the $n$ 's varied between 14 and 17 .

\subsection{Statistical tests}

Because most variables were not normally distributed and could not be normalized, non-parametric Mann-Whitney $U$-tests were used for the statistical analyses.

\section{Results}

\subsection{Preliminary analyses}

\subsubsection{High and low prenatal cortisol groups}

The time of saliva collection in pregnancy weeks 37 and 38 was different for each individual mother: varying between 0730 and $1000 \mathrm{~h}$, with each woman taking the first sample at awakening, and the second sample as a control at the same time of day a week later. The cortisol values varied between 13 and 46 nmol/1, mean: 25.3, S.D.: 6.7. Due to 
the circadian rhythm, cortisol levels decrease during the morning hours [25]. In our data, the Pearson's correlation between the sampling time and the mean cortisol levels was not significant $(r=-0.28, p=0.14)$. However, because the study population was small in number and the cortisol levels did display a clear negative trend with the time of morning at which the saliva samples were taken, we corrected the values for circadian effects on the cortisol levels. This was done as follows. For each subject both prenatal samples did not differ significantly from each other, and therefore the mean value was calculated. These mean values were then regressed against the time of day at which the women had sampled saliva. The residuals were calculated and the median of these residuals was used for dividing the subjects into the high (residual falls above the median, $n=8$ ) and low (residual falls below the median, $n=9$ ) prenatal cortisol groups.

\subsubsection{Prenatal cortisol groups and duration of pregnancy}

As shown above, the group of 17 mothers was divided into high and low prenatal cortisol groups on the basis of their prenatal cortisol values, of which there were only two samples. To check the validity of this subdivision, the gestation length was compared between the two groups. As mentioned in Section 1, prenatal stress has often been found to be associated to shorter pregnancies. Supporting the subdivision, the higher cortisol group of women was found to have a strong tendency for shorter pregnancies than the lower cortisol group (Mann-Whitney test, $z=-1.4$, one-tailed $p=0.08$ ). The pregnancies of low prenatal cortisol mothers were almost 6 days longer than the pregnancies of high prenatal cortisol mothers. The lower cortisol group delivered at a mean of 3.6 days later than the due date, while the higher cortisol group delivered at a mean of 1.7 days earlier than the due date.

\subsubsection{Data reduction}

In order to smooth out the effects of individual bathing sessions, the means of the behaviors were calculated over the following baths: weeks 1 and 3, weeks 5 and 7, and

Table 1

Behaviors in which significant differences were found between the infants from mothers with higher cortisol levels and those from mothers with lower cortisol levels

\begin{tabular}{|c|c|c|c|c|c|}
\hline \multirow[t]{2}{*}{ Behavior } & \multirow[t]{2}{*}{ Weeks } & \multicolumn{2}{|l|}{ Mean rank } & \multirow[t]{2}{*}{$Z$} & \multirow[t]{2}{*}{$P$ one-tailed } \\
\hline & & High cortisol & Low cortisol & & \\
\hline \multirow[t]{3}{*}{ Crying } & $1-3$ & 10.75 & 6.25 & -1.89 & 0.03 \\
\hline & $5-7$ & 11.13 & 5.88 & -2.22 & 0.01 \\
\hline & $18-20$ & 8.75 & 8.25 & -0.23 & 0.41 \\
\hline \multirow[t]{3}{*}{ Fussing } & $1-3$ & 11.25 & 5.75 & -2.31 & 0.01 \\
\hline & $5-7$ & 10.00 & 7.00 & -1.26 & 0.10 \\
\hline & $18-20$ & 10.88 & 6.13 & -1.99 & 0.02 \\
\hline \multirow[t]{3}{*}{ Negative facial expression } & $1-3$ & 10.75 & 6.25 & -1.89 & 0.03 \\
\hline & $5-7$ & 11.25 & 5.75 & -2.31 & 0.01 \\
\hline & $18-20$ & 10.69 & 6.31 & -1.84 & 0.03 \\
\hline \multirow[t]{3}{*}{ No vocalization } & $1-3$ & 5.38 & 11.63 & -2.63 & 0.004 \\
\hline & $5-7$ & 6.00 & 11.00 & -2.10 & 0.02 \\
\hline & $18-20$ & 7.50 & 9.50 & -0.84 & 0.20 \\
\hline
\end{tabular}


weeks 18 and 20. In this way, the amount of data points was reduced to three: two 'neonatal' assessments and one assessment around 4-5 months of age.

\subsubsection{Influence of parity on maternal cortisol and behavior}

Primiparous subjects $(n=7)$ were compared to multiparous subjects $(n=10)$ in order to see whether they differed in their prenatal cortisol levels and/or their behavior during the bathing session. No significant differences were found between the groups with respect to any of the studied variables. Parity was therefore no longer taken into account in subsequent analyses.

\subsection{Prenatal cortisol and maternal behavior}

To find out whether the mothers with higher cortisol levels during pregnancy $(n=8)$ behaved differently than those with lower cortisol levels $(n=9)$, Mann-Whitney tests

\section{a)}

fussing

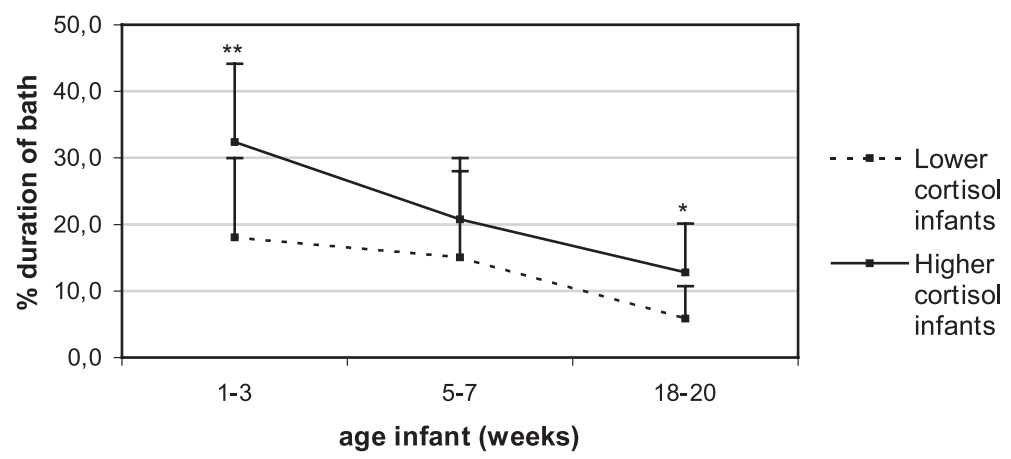

b)

\section{crying}

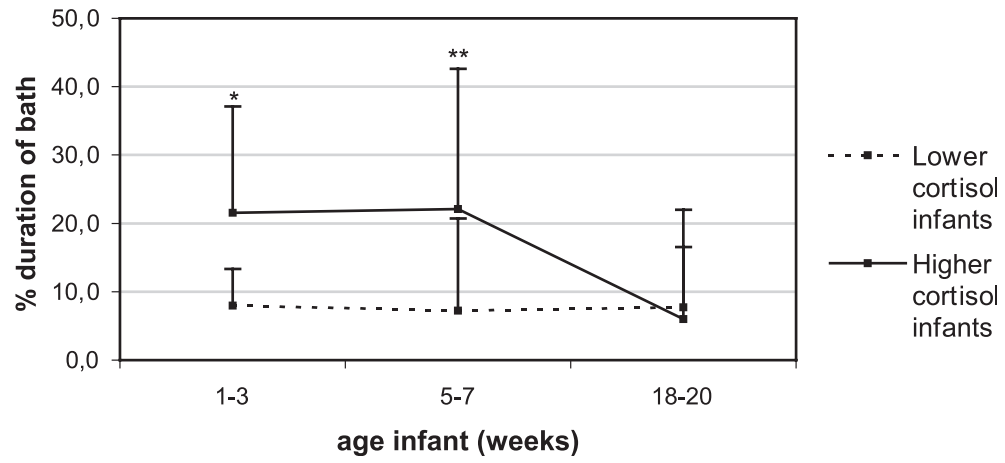

Fig. 2. $(a-d)$ Behavioral differences between the groups of infants during the bath session: mean percentages of time displaying the behavior at the three ages. Error bars are standard deviations of the mean; significancies are indicated as follows: ${ }^{*} p \leq 0.01,{ }^{*} p \leq 0.05$. 
c)

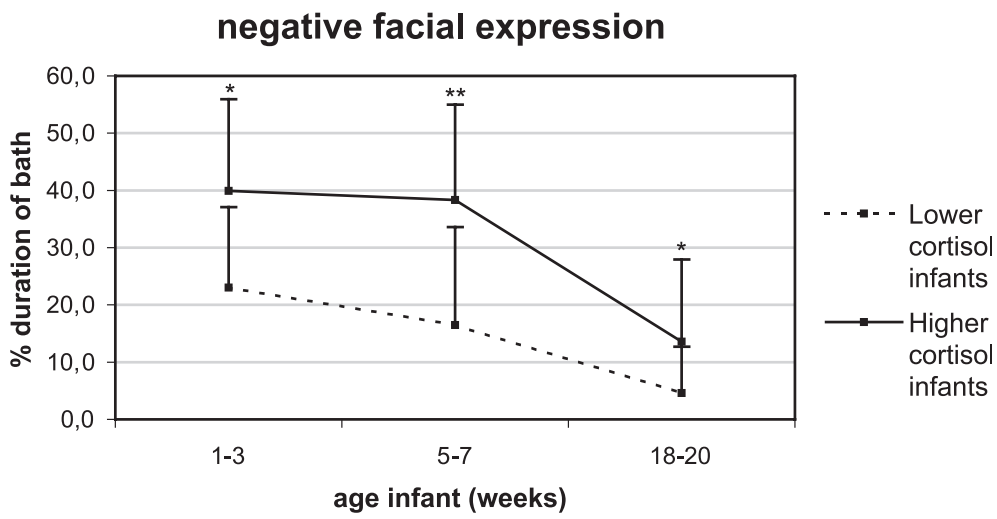

d)

no vocalization

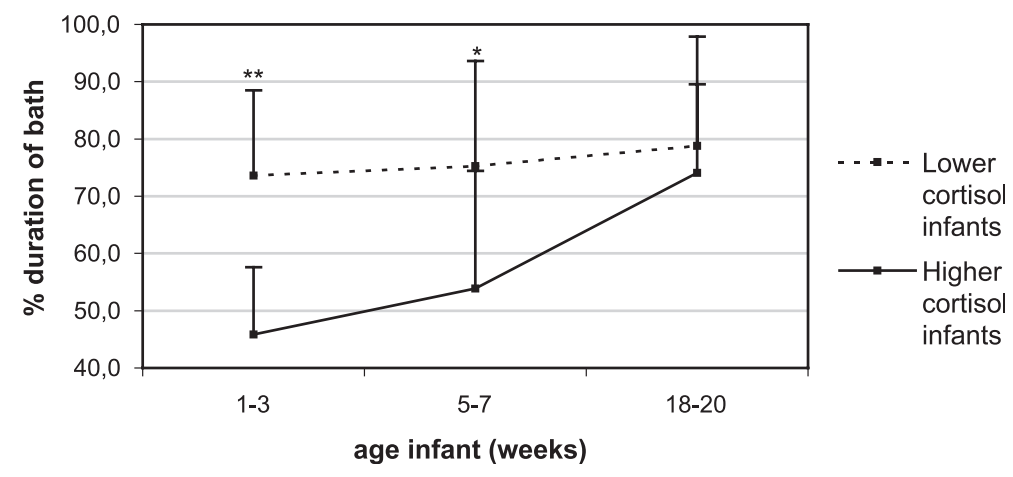

Fig. 2 (continued).

were carried out on all the maternal behaviors, for the neonatal period and the $4-5$-month period.

Nine behaviors of the mothers were analyzed (see Ethogram above) and only one significant difference was found: 'kissing' occurred significantly more often in the high prenatal stress group than in the low prenatal stress group. This was the case in weeks $1-3$ $(Z=-2.42$, two-tailed $p=0.01)$ and $5-7(Z=-2.10$, two-tailed $p=0.04)$, and in weeks $18-20$ a tendency for significance was found $(Z=-1.85$, two-tailed $p=0.06)$.

\subsection{Prenatal cortisol and infant behavior}

The behavior of the infants born from mothers with higher cortisol levels was compared to that of infants born from mothers with lower cortisol levels. Out of the 15 behaviors analyzed, 4 were significantly different between the groups: 'crying', 'fussing', 'negative facial expression' and 'no vocalization' (see Table 1 and Fig. $2 \mathrm{a}-\mathrm{d}$ ). The difference in 'no vocalization' is due to the infants in the higher cortisol group crying and fussing more, as 
Table 2

Differences between the infants from mothers with higher cortisol levels and those from mothers with lower cortisol levels with respect to the ICQ temperamental categories

\begin{tabular}{|c|c|c|c|c|c|}
\hline \multirow[t]{2}{*}{ Temperament category } & \multirow[t]{2}{*}{ Week } & \multicolumn{2}{|l|}{ Mean rank } & \multirow[t]{2}{*}{$Z$} & \multirow[t]{2}{*}{$P$} \\
\hline & & High cortisol & Low cortisol & & \\
\hline \multirow[t]{2}{*}{ Emotion } & 7 & 10.00 & 6.25 & -1.63 & 0.05 \\
\hline & 18 & 9.06 & 7.94 & -0.47 & 0.32 \\
\hline \multirow[t]{2}{*}{ Activity } & 7 & 11.07 & 5.31 & -2.52 & 0.006 \\
\hline & 18 & 9.81 & 7.19 & -1.12 & 0.13 \\
\hline \multirow[t]{2}{*}{ Adaptation } & 7 & 9.07 & 7.06 & -0.88 & 0.19 \\
\hline & 18 & 8.38 & 8.63 & -0.11 & 0.46 \\
\hline
\end{tabular}

no significant differences were found in the amount of positive or neutral vocalizations ('happy').

As can be seen in the table and figure, the infants from the higher cortisol group were more irritable and showed more negative affect during the bathing sessions than those from the lower cortisol group.

\subsection{Prenatal cortisol and infant temperament}

The 7- and 18-week temperament scores of infants from the high prenatal cortisol group were compared to the scores of infants from the low prenatal cortisol group. Higher scores mean more difficulties in that category of temperament.

In the categories Emotion and Activity, the infants from the high prenatal cortisol group had significantly higher scores than the infants from the low prenatal cortisol group (see results of one-tailed Mann-Whitney $U$-tests in Table 2). In both cases, the differences could be seen in week 7, but in week 18 they had disappeared. No significant differences were found in the category Adaptation.

Also, different developmental trends were displayed by the groups of mothers over all three temperamental categories: while the high prenatal cortisol mothers reported less difficulties at 18 weeks, the low prenatal cortisol mothers reported more difficulties at that age.

\section{Discussion}

In this study, higher prenatal cortisol levels at the end of pregnancy were related to more crying, fussing and negative facial expressions in the infant during a series of routine mother-infant interactions. Supporting the behavioral observations, maternal reports on temperament also showed these infants to have more difficult behavior: they had higher scores on emotion and activity. The differences between the infants were strongest in the neonatal period. At 4/5 months of age, most significant differences had disappeared, although the infants born from mothers with higher cortisol levels still fussed more during the interactions, and also had a tendency to spend more time displaying a negative facial expression. 
At 18 weeks postnatally, the maternal reports on temperament in both groups of mothers grew more similar to each other, making the earlier significant differences disappear. While the mothers from the higher prenatal cortisol group clearly reported less difficult behavior, the mothers from the lower prenatal cortisol group reported more difficult behavior in their infants. This was so for all three temperamental categories.

At least some behavioral changes could be observed between the neonatal and 4/5month period in how the infants reacted to the bath situation. Supporting the reports of the mothers in the high cortisol group, the infants became less irritable during the baths. This contradicts, however, the reports of increases in difficulty of the mothers in the lower cortisol group. Therefore, these changes in temperament reports over age are probably due to a combination between actual changes in behavior in the infants and changes in how the mothers experience their infants' behavior.

The fact that the infants in general showed less negative affect during the baths at 1820 weeks of age (Fig. 2a-d) could be due to the infants' changing appraisal of the bath situation. It is possible that while in the neonatal period the bath was appraised by the infants as a (mildly) stressful situation, they later on commenced to enjoy the bath session. Also, positive experiences during the first months of life with caregiver interactions and contingency of caregiver behavior could explain at least in part the decreases in negative affect for this group of infants.

Interestingly, although the group as a whole showed decreases in negative affect, the infants from mothers with higher cortisol still displayed significantly more fussing and negative facial expressions at $4 / 5$ months than the infants from lower cortisol mothers. Only the difference in crying (and possibly consequently, the difference in time spent silent) disappeared. That these differences in irritability are still present at $4 / 5$ months of age could point at the presence of programming effects of cortisol during pregnancy. Therefore, situations, which at this age would be generally appraised as stressful (i.e. a painful stimulus such as an inoculation), might uncover still larger differences in behavior between the groups of infants.

When the behavior of the mothers with higher cortisol values was compared to that of those with lower cortisol, the groups were found to greatly resemble each other in how they interacted with their infants. Out of nine observed behaviors, only 'kissing' was performed more frequently by the higher cortisol mothers than by the lower cortisol mothers. A possible explanation for this only difference is that because the infants of the higher cortisol group cried/fussed more, their mothers kissed them more frequently in an attempt at soothing them. The mothers would react to their infants' crying/fussing bouts by kissing them. But post hoc lag sequential analyses of patterns of mother-infant interactions showed no differences in behavioral patterns between both groups of mother-infant pairs, making this explanation not very plausible. Although this one difference between the mothers is difficult to explain, the main finding is that the mothers acted very similarly towards their infants. The question remains, however, whether the bath situation is representative for other types of interactions between the mother and her infant. More behavioral differences between the mothers could for example become apparent in a 'freer' situation, such as in a purely playful interaction.

When interpreting the results presented in this paper, it is important to keep in mind that they are the products of a relatively small study, with several limitations. Firstly, the 
number of subjects was small and it remains to be seen whether the results can be replicated in a larger population. Secondly, the prenatal predictor was limited to two samples of late pregnancy morning cortisol. Future studies could be enriched by adding cortisol circadian rhythm data in which, for example, the curve form or the evening cortisol is used as predictor. Other data that would possibly give more weight to the prenatal information are questionnaires on stressors, both daily hassles and life events, and on anxiety and perceived stress. It would also be interesting to follow infants for a longer period and investigate whether the effects found persist into childhood. However, it is important to stress that although the data pertain a small study with limited statistical power, its intensive longitudinal nature, together with the fact that the relations found between prenatal cortisol and infant behavior were in the expected direction, make the results in our view extremely interesting. Especially so, because both the behavioral observations as the maternal reports show that infants from higher or lower pregnancy cortisol behave differently during their first few months of life.

\section{Acknowledgements}

The authors wish to thank the families that participated in the study and also Birgitte de Vries for helping to collect the data. This research was supported by the Netherlands Organization for Scientific Research (NWO), grant number 575-25-009.

\section{References}

[1] Stott DH. Follow-up study from birth of the effects of prenatal stresses. Dev Med Child Neurol 1973;15:770-87.

[2] Barker DJP. Mothers, babies, and disease in later life. London: BMJ Publishing Group; 1994.

[3] Selten JP, van der Graaf Y, van Duursen R, Gispen-de Wied CC, Kahn RS. Psychotic illness after prenatal exposure to the 1953 Dutch Flood Disaster. Schizophr Res 1999;35:243-5.

[4] Checkley S. The neuroendocrinology of depression and chronic stress. Br Med Bull 1996;52:597-617.

[5] Hedegaard M, Henriksen TB, Sabroe S, Secher NJ. Psychological distress in pregnancy and preterm delivery. BMJ 1993;307:235-9.

[6] Copper RL, Goldenberg RL, Das A, Elder N, Swain M, Norman G, et al. The preterm prediction study: maternal stress is associated with spontaneous preterm birth at less than thirty-five weeks' gestation. Am J Obstet Gynecol 1996;175:1286-92.

[7] Nordentoft M, Lou HC, Hansen D, Nim J, Pryds O, Rubin P, et al. Intrauterine growth retardation and premature delivery: the influence of maternal smoking and psychosocial factors. Am J Public Health 1996;86:347-54.

[8] Seck1 JR. Glucocorticoids, feto-placental 11 beta-hydroxysteroid dehydrogenase type 2, and the early life origins of adult disease. Steroids 1997;62:89-94.

[9] Ponirakis A, Susman EJ, Stifter CA. Negative emotionality and cortisol during adolescent pregnancy and its effects on infant health and autonomic nervous system reactivity. Dev Psychobiol 1998;33:163-74.

[10] Hansen D, Lou HC, Olsen J. Serious life events and congenital malformations: a national study with complete follow-up. Lancet 2000;356:875-80.

[11] Mulder EJH, Robles de Medina PG, Huizink AC, Van den Bergh BRH, Buitelaar JK, Visser GHA. Prenatal maternal stress: effects on pregnancy and the (unborn) child. Early Hum Dev 2002;70:3-14.

[12] Gitau R, Fisk NM, Glover V. Maternal stress in pregnancy and its effect on the human foetus: an overview of research findings. Stress 2001;4:195-203. 
[13] Wadhwa PD, Glynn L, Hobel CJ, Garite TJ, Porto M, Chicz-DeMet A, et al. Behavioral perinatology: biobehavioral processes in human fetal development. Regul Pept 2002;108:149-57.

[14] Susman EJ, Schmeelk KH, Ponirakis A, Gariepy JL. Maternal prenatal, postpartum, and concurrent stressors and temperament in 3-year-olds: a person and variable analysis. Dev Psychopathol 2001;13:629-52.

[15] Van Goozen SHM, Matthys W, Cohen Kettenis PT, Buitelaar JK, Van Engeland H. Hypothalamic-pituitary-adrenal axis and autonomic nervous system activity in disruptive children and matched controls. J Am Acad Child Adolesc Psychiatry 2000;39:1438-45.

[16] Pajer K, Gardner W, Rubin RT, Perel J, Neal S. Decreased cortisol levels in adolescent girls with conduct disorder. Arch Gen Psychiatry 2001;58:297-302.

[17] McBurnett K, Lahey BB, Rathouz PJ, Loeber R. Low salivary cortisol and persistent aggression in boys referred for disruptive behavior. Arch Gen Psychiatry 2000;57:38-43.

[18] O'Connor TG, Heron J, Golding J, Beveridge M, Glover V. Maternal antenatal anxiety and children's behavioural/emotional problems at 4 years. Report from the Avon Longitudinal Study of Parents and Children. Br J Psychiatry 2002;180:502-8.

[19] Huizink AC, Robles de Medina PG, Mulder EJ, Visser GH, Buitelaar JK. Psychological measures of prenatal stress as predictors of infant temperament. J Am Acad Child Adolesc Psychiatry 2002;41:1078-85.

[20] Huizink AC, Robles de Medina PG, Mulder EJ, Visser GH, Buitelaar JK. Psychosocial and endocrinologic measures of prenatal stress as predictors of mental and motor development in infancy. J Child Psychology Psychiatry 2003 (in press).

[21] Graham YP, Heim C, Goodman SH, Miller AH, Nemeroff CB. The effects of neonatal stress on brain development: implications for psychopathology. Dev Psychopathol 1999;11:545-65.

[22] Meijer A. Child psychiatric sequelae of maternal war stress. Acta Psychiatr Scand 1985;72:505-11.

[23] Cohen J. Coefficient of agreement for nominal scales. Educ Psychol Meas 1960;20:37-46.

[24] Bates JE, Freeland CA, Lounsbury ML. Measurement of infant difficultness. Child Dev 1979;50:794-803.

[25] Kirschbaum C, Hellhammer DH. Salivary cortisol in psychobiological research: an overview. Neuropsychobiology 1989;22:150-69. 